\title{
LA FORMACIÓN INICIAL DEL PROFESORADO DE SECUNDARIA EN LA UNIÓN EUROPEA
}

\author{
Initial teacher training for secondary education in the \\ European Union
}

\author{
Jesús Manso y Javier M. Valle *
}

\section{RESUMEN}

La creciente presencia de las medidas y propuestas desde la Unión Europea en materia de formación inicial del profesorado parece cada vez más evidente. Sin embargo, parece necesario identificar en qué grado existe una armonización entre los países en esta cuestión. Con ello, pretendemos identificar algunas tendencias que nos ayuden a reflexionar sobre las políticas educativas de la Unión Europea y de las concreciones de países concretos. Para la consecución de este objetivo general utilizaremos la metodología comparada. Los resultados y conclusiones del estudio muestran las tendencias que podrían ser susceptibles de integrarse en las políticas educativas nacionales con las necesarias adaptaciones en el pleno respeto a las propias características y tradiciones educativas de cada país.

PALABRAS CLAVE: formación inicial, profesorado, política educativa, Unión Europea, educación supranacional.

\footnotetext{
*Universidad Autónoma de Madrid (España).
} 


\section{$\underline{\text { ABSTRACT }}$}

The measures and proposals of the European Union in the field of initial teacher education are increasingly evident. However, it is necessary to identify the stage of harmonization on this issue between countries. Thus, we aim to identify some trends to help us to reflect on the initial teacher training policies of the European Union and individual countries. To achieve this overall objective we will use the comparative method. The results and conclusions of the study show trends that could be susceptible to integrate into national education policies with the necessary adaptations that they respect the characteristics and educational traditions of each country.

KEY WORDS: initial teacher education, education policy, European Union, supranational education.

$* * * * *$

\section{INTRODUCCIÓN}

El análisis de la evolución histórica de la Unión Europea permite identificar su interés creciente a la hora de definir políticas en el ámbito educativo (VALLE, 2009). Así, numerosos estudios ponen de manifiesto una evolución creciente de las denominadas políticas educativas de la Unión Europea, especialmente a partir del Consejo de Lisboa del año 2000 (PHILIPS y ERTL, 2003; ERTL, 2006; FIELD y MURPHY, 2006).

A este respecto, resulta pertinente señalar que estas propuestas están cada vez más presentes en las políticas concretas de los Estados miembros, quienes las aceptan y ponen en marcha, adaptándolas a sus realidades nacionales. Existen recientes evidencias empíricas al respecto, como por ejemplo las políticas relacionadas con el Espacio Europeo de Educación Superior (EEES), el aprendizaje permanente -a lo largo de la vida- o las Competencias Clave (KWIEK, 2004; TUSCHLING y ENGEMANN, 2006; DEHMEL, 2006).

En este contexto, el profesorado, como elemento esencial en la mejora de la educación, ha estado presente en estas políticas especialmente desde el Acta Única de 1986. Como indica Egido (1996), mediante este tratado internacional -firmado por los doce Estados miembros que en aquel momento configuraban la denominada Comunidad Europea- las políticas educativas sobre el docente comienzan a introducirse con mayor fuerza, pero siempre como medio para objetivos más amplios. Muchas de las medidas asumidas en aquel documento tenían una relación muy directa con la formación de profesorado, llegando incluso a plantearla como una necesidad.

Este artículo pretende conocer cuáles son las principales tendencias actuales en la formación inicial del profesorado emanadas desde la Unión Europea como realidad supranacional. Ello, con vistas a reflexionar sobre la organización y orientaciones que actualmente existen en nuestro contexto nacional. 
Para la consecución de este objetivo, hemos optado por el método comparado aplicado sobre cuatro países de la Unión: Alemania, Finlandia, Francia e Inglaterra. El criterio principal para la selección de estas unidades de comparación ha sido su ubicación en tradiciones educativas diversas (centroeuropea, nórdica, napoleónica y anglosajona, respectivamente) pero comparables ${ }^{1}$. Las principales fuentes utilizadas para su estudio han sido los propios documentos que cada uno de los países ha generado para la red Eurydice (2010a, 2010b, 2010c, 2010d) y el último informe de esta red europea, Key data (EURYDICE, 2012).

\section{LA FORMACIÓN INICIAL DEL PROFESORADO EN LA POLÍTICA DE LA UNIÓN EUROPEA}

De la revisión de toda la documentación europea en materia de formación inicial del profesorado, el primer aspecto a destacar es el hecho de que hasta la Estrategia de Lisboa ${ }^{2}$ (CONSEJO EUROPEO, 2000) las políticas en esta materia han tenido un carácter puntual y subsidiario frente a otros programas u objetivos específicos. Hasta ese momento, es posible encontrar fuentes que indican la relevancia de la formación inicial, pero siempre puesta al servicio de otras políticas educativas ${ }^{3}$. La formación del docente en general, y su formación inicial en particular, comienzan a adquirir mayor relevancia a partir de la Estrategia de Lisboa, y con su incorporación entre los Objetivos Precisos propuestos en 2001 para los sistemas educativos.

En el Consejo Europeo de Lisboa la Unión Europea adoptó un objetivo estratégico importante: antes de que concluyera 2010 la Unión tenía que convertirse en la economía, basada en el conocimiento, más competitiva y dinámica del mundo, capaz de crecer económicamente de manera sostenible con más y mejores empleos y con mayor cohesión social (parágrafo I.5). Como consecuencia de este encuentro, el Consejo de Educación emprende una reflexión general sobre los futuros Objetivos Precisos de los sistemas educativos: se dedica a definir intereses y prioridades comunes y que respeten, al mismo tiempo, la diversidad entre los países. De este proceso se deriva el documento denominado Informe del Consejo de Educación, del 12 de febrero de 2001, dirigido al Consejo Europeo sobre los futuros objetivos precisos de los sistemas de educación y formación (CONSEJO DE EDUCACIÓN, 2001) que será en el que por primera vez aparecen los que denominarán Objetivos Precisos. En este documento se establecían tres objetivos generales y trece objetivos concretos (los denominados propiamente "precisos"). El primero que se incluye es Mejorar la educación y la formación de profesores y formadores. El texto que los desarrolla indica:

\footnotetext{
${ }^{1}$ Los resultados del estudio comparado se presentan en el segundo epígrafe de este trabajo. Por motivos de espacio no será posible explicitar todos los elementos propios de las fases descriptivas e interpretativas, sino que se ha optado por presentar únicamente la información yuxtapuesta, el resultado de la comparación y las conclusiones prospectivas.

${ }^{2}$ La Estrategia de Lisboa es un concepto que no responde a ningún programa, acción o documento concretos de la Unión Europea sino al proceso de actuaciones, globales y sucesivas, consecuencia del Consejo Europeo de Lisboa, celebrado los días 23 y 24 de marzo del año 2000.

${ }^{3}$ Ejemplo de ello serían documentos relacionados con programas concretos (especialmente Lingua -Consejo, 1998a- y Tecnologías de la Información y la Comunicación -Consejo, 1996-) y otros destinados a colectivos minoritarios (gitanos - Consejo, 1989b-, niños y jóvenes minusválidos - Consejo, 1990a- o cuestiones de género - Consejo, 1990b-), entre otros.
} 
"Uno de los principales retos a los que habrán de enfrentarse los sistemas de educación y formación en los próximos diez años es el de mejorar la educación y la formación iniciales y durante el servicio de los profesores y los formadores, de manera que sus conocimientos y capacidades respondan a los cambios y a las expectativas de la sociedad, y se adapten a la variedad de grupos que ellos educan y forman" (CONSEJO DE EDUCACIÓN, 2001: 8).

Como consecuencia del trabajo realizado por el equipo de los Objetivos Precisos, creció considerablemente la importancia concedida en los documentos sobre política educativa de la Unión Europea a la formación del profesorado. En este contexto, en 2005 la Comisión Europea publicó el que es, en nuestra opinión, el documento más importante al respecto de las competencias docentes. En él se definen cuáles son los principios europeos comunes sobre su perfil competencial (EUROPEAN COMMISSION, 2005).

Los principios comunes que se estiman de la función docente se sintetizan en los cuatro siguientes: en primer lugar, que sea una profesión que exija una formación de nivel de posgrado en instituciones de Educación Superior; un segundo elemento fundamental es el de entender la docencia como una profesión ubicada en el contexto del aprendizaje permanente; en tercer lugar, se plantea que la docencia debe entenderse como una profesión móvil, esto es, dinámica y sujeta al enriquecimiento a través de estancia de los docentes en otras realidades educativas; y, por último, el documento sostiene que la profesión docente debe estar basada en la asociación y en el trabajo en red de centros y profesores de diferentes lugares europeos.

Todos estos principios se presentan como el marco necesario para comprender que los profesores deben trabajar eficazmente en las siguientes tres áreas que la Unión Europea propone que deben favorecerse desde los programas de formación del profesorado de los Estados miembros:

- Trabajar con otros: iguales, estudiantes, familias...

- Trabajar con el conocimiento, la tecnología y la información.

- Trabajar con y en la sociedad: a nivel local, regional y nacional.

Dos años más tarde, en 2007, se publica el documento sobre La Calidad de la Formación del Profesorado. En él, la formación inicial y la inserción en el lugar de trabajo adquieren una mayor relevancia. De esta manera, la Unión Europea propone ver el desarrollo de competencias profesionales sobre la continuidad de la vida profesional.

Este documento supuso la justificación necesaria para proponer un trabajo sobre competencias docentes a nivel europeo. Así, la Unión Europea hizo pública una convocatoria para la realización de un proyecto de investigación con el objetivo de determinar el que podría ser un curriculum de la formación del profesorado compartido por el conjunto de los países de la Unión. Finalmente sería el Finnish Institute for Educational Research (FIER) quien llevara a término esta investigación. El producto final de este trabajo se sintetiza en ocho ámbitos sobre los que se proponen que giren las competencias profesionales de los docentes. Estos se muestran en la figura 1 y pueden ser considerados como el perfil competencial del docente europeo. 


\section{Figura 1. Competencias profesionales del profesorado europeo actual}
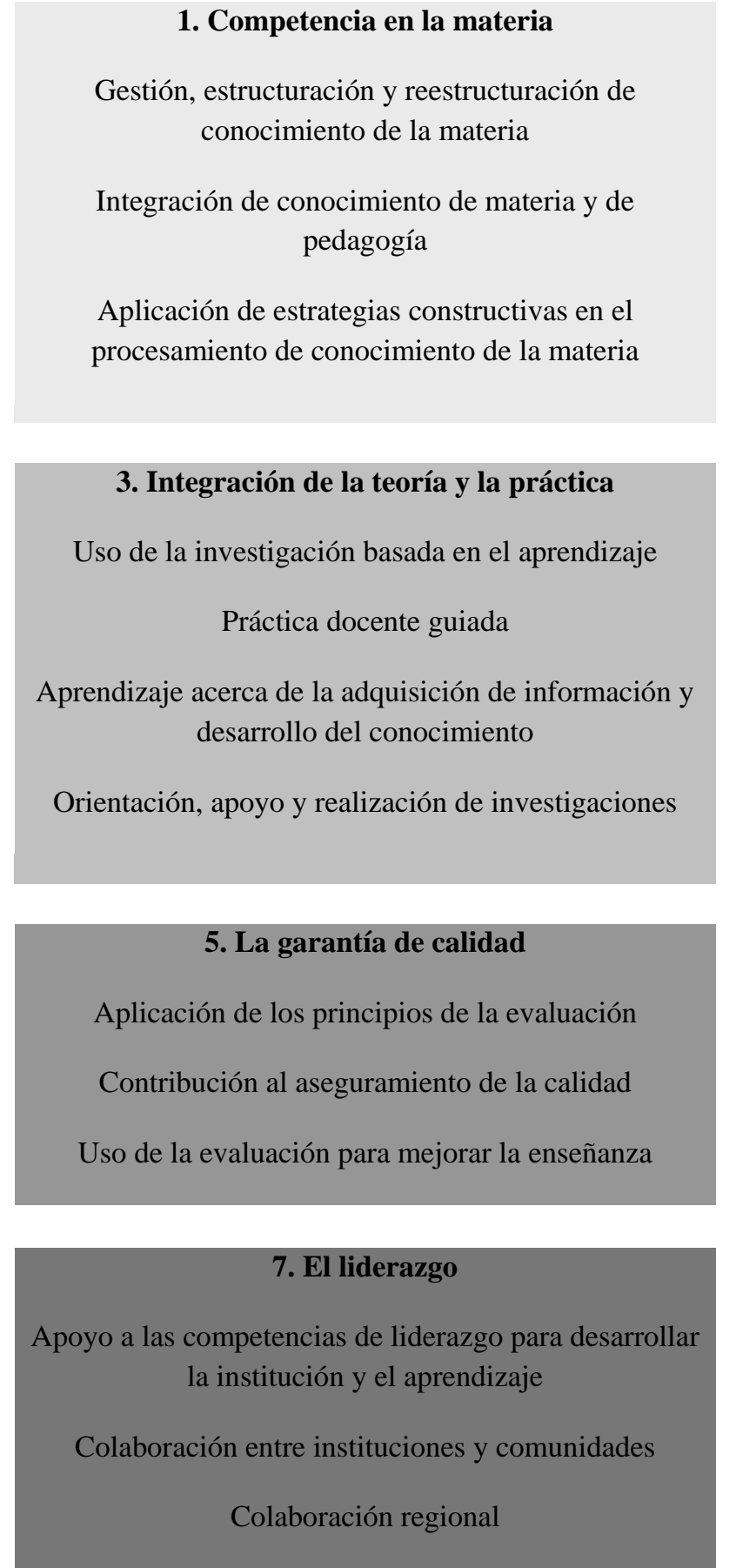

Fuente: elaboración propia a partir de FIER (2009).

\section{Competencias pedagógicas}

Empleo de una gama de estrategias de enseñanza

Utilización de diversos métodos de enseñanza

Desarrollo socio-emocional y moral (estudiantes)

Enseñanzas heterogéneas clases

Orientación y apoyo a los estudiantes

\section{Cooperación y colaboración}

Entre los estudiantes, colegas, padres y escuelas

Trabajar eficazmente con la comunidad local

Apoyar las habilidades de comunicación

Uso de métodos de aprendizaje colaborativos

Promoción de un ambiente escolar respetuoso
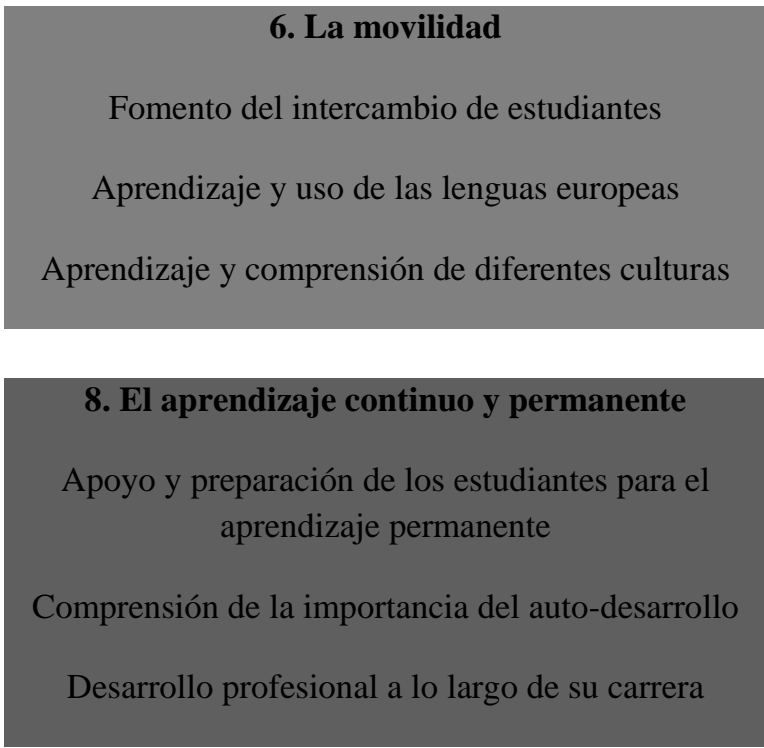

A pesar de todos estos esfuerzos, la relevancia otorgada a la formación inicial del profesorado, desde los documentos oficiales de la política educativa de la Unión Europea, parece haberse reducido considerablemente. Existen dos factores principales que podrían explicar este hecho. Por un lado, la actual situación económica, que supone poner el acento de las políticas de la 
Unión Europea en otras actuaciones consideradas preferentes; más cercanas a preocupaciones económicas que socioeducativas. Y, en segundo lugar, el inicio de un nueva estrategia de acción (Educación y Formación 2020), que supone una reformulación de los objetivos de la política educativa de la Unión Europea.

Como se puede comprobar en la documentación, las propuestas de las Unión Europea en materia de formación inicial deben considerarse como principios rectores y teóricos para que los Estados miembros puedan tener en consideración a la hora del desarrollo normativo en esta materia. Por ello, por ejemplo, no se muestran diferencias entre las sugerencias destinadas a profesores de etapas educativas diferentes. Además, hay que tener en cuenta que siguen siendo los diferentes países los que determinan completamente cómo organizar la formación inicial de su profesorado. A continuación, se abordarán las principales características, en perspectiva comparada, de los modelos de formación inicial del profesorado de Educación Secundaria (FIPES) en Alemania, Finlandia, Francia e Inglaterra.

\section{LA FORMACIÓN INICIAL DEL PROFESORADO DE EDUCACIÓN SECUNDARIA (FIPES) EN PAÍSES EUROPEOS ${ }^{4}$}

Hasta el momento hemos podido comprobar la existencia de una política educativa de la Unión Europea que pone de manifiesto la importancia de la formación inicial del profesorado en las últimas décadas. Sin embargo, la armonización que en otros ámbitos educativos se está produciendo (como, por ejemplo, el de la Educación Superior o las Competencias Clave), no se ha visto, al menos de momento, tan influida en el caso de la formación inicial del profesorado.

Así, el primer tema que se pone de manifiesto, fruto del estudio realizado sobre los países seleccionados, es que existe una amplia diversidad de maneras de organizar la FIPES. Sin embargo, un segundo aspecto relevante es la convergencia entre los países seleccionados sobre el hecho de que su incorporación al Espacio Europeo de Educación Superior haya supuesto modificaciones en los sistemas de FIPES. Así lo atestiguan las nuevas normativas de Alemania (2004), Finlandia (2005), España (2009) y Francia (2010).

A continuación pasamos a desarrollar aspectos más específicos que definen las principales convergencias y divergencias de los países seleccionados y que definen cuatro modelos distintos de organizar y entender la FIPES.

Tabla 1. Modelos de FIPES de países europeos

\begin{tabular}{|c|c|c|c|c|c|}
\cline { 2 - 5 } \multicolumn{1}{c|}{} & Alemania & Finlandia & Francia & Inglaterra & España \\
\hline $\begin{array}{c}{ }^{\text {(a) }} \begin{array}{c}\text { Marco institucional } \\
\text { principal en el que se } \\
\text { desarrolla el programa }\end{array} \\
\text { Universidades }\end{array}$ & Universidades & $\begin{array}{c}\text { Universidades } \\
\text { y IUFM }\end{array}$ & $\begin{array}{c}\text { Instituciones } \\
\text { acreditadas } \\
\text { por la TA }\end{array}$ & Universidades \\
\hline
\end{tabular}

\footnotetext{
${ }^{4}$ Se ha optado por definir el profesorado de Educación Secundaria por considerar que es esta etapa la que incluye mayores controversias y, por tanto, la que ofrece un enriquecimiento mayor de las conclusiones.
} 


\begin{tabular}{|c|c|c|c|c|c|c|}
\hline \multicolumn{2}{|c|}{$\begin{array}{l}\text { (b) Nivel de certificación } \\
\text { mínima obtenida } \\
\text { (duración mínima total) }\end{array}$} & $\begin{array}{c}\text { Máster } \\
\text { años })\end{array}$ & $\begin{array}{l}\text { Máster } \\
\text { (5 años ó } \\
\text { 3+2 años) }\end{array}$ & $\begin{array}{c}\text { Máster } \\
(3+2 \text { años })\end{array}$ & $\begin{array}{c}\text { QTS } \\
\text { Grado } \\
\text { (3/4 años) }\end{array}$ & $\begin{array}{c}\text { Máster } \\
(4+1 \text { años })\end{array}$ \\
\hline \multicolumn{2}{|c|}{$\begin{array}{l}{ }^{(b)} \text { Modelo estructural del } \\
\text { programa de FIPES }\end{array}$} & Concurrente & Mixto & Consecutivo & Mixto & Consecutivo \\
\hline \multicolumn{2}{|c|}{$\begin{array}{l}\text { (a) \% formación didáctico- } \\
\text { pedagógica mínima }\end{array}$} & $57 \%$ & $21,9 \%$ & --- & $25 \%$ & $20 \%$ \\
\hline \multirow{2}{*}{$\begin{array}{l}{ }^{(b)} \text { Requisitos } \\
\text { acceso al } \\
\text { programa } \\
\text { formación } \\
\text { didáctico- } \\
\text { pedagógica }\end{array}$} & $\begin{array}{c}\text { Conforme } \\
\text { al modelo } \\
\text { concurrente }\end{array}$ & $\begin{array}{l}\text { Hochschulreife } \\
\text { y Abitur }\end{array}$ & $\begin{array}{c}\text { Examen, } \\
\text { prueba aptitud } \\
\text { y entrevista... }\end{array}$ & --- & $\begin{array}{c}\text { Determina la } \\
\text { institución } \\
\text { formadora }\end{array}$ & --- \\
\hline & $\begin{array}{c}\text { Conforme } \\
\text { al modelo } \\
\text { consecutivo }\end{array}$ & --- & $\begin{array}{c}\text { Expediente } \\
\text { académico y } \\
\text { prueba aptitud }\end{array}$ & --- & $\begin{array}{l}\text { Determina la } \\
\text { institución } \\
\text { formadora }\end{array}$ & $\begin{array}{c}\text { Grado + B1 de } \\
\text { lengua } \\
\text { extranjera }\end{array}$ \\
\hline \multicolumn{2}{|c|}{$\begin{array}{l}{ }^{\text {(b) }} \text { Requisito de acceso a la } \\
\text { profesión (centros } \\
\text { públicos) }\end{array}$} & $\begin{array}{l}\text { Marcado por la } \\
\text { fase de } \\
\text { cualificación } \\
\text { en el puesto de } \\
\text { trabajo }\end{array}$ & $\begin{array}{l}\text { Contratación } \\
\text { local o por los } \\
\text { centros }\end{array}$ & $\begin{array}{l}\text { Examen de } \\
\text { reclutamiento } \\
\text { estatal }\end{array}$ & $\begin{array}{c}\text { Marcado por } \\
\text { la fase de } \\
\text { cualificación } \\
\text { en el puesto } \\
\text { de trabajo }\end{array}$ & $\begin{array}{c}\text { Examen de } \\
\text { oposiciones } \\
\text { con directrices } \\
\text { estatales }\end{array}$ \\
\hline \multicolumn{2}{|c|}{$\begin{array}{c}\text { (b) Fase de cualificación en } \\
\text { el lugar de trabajo - } \\
\text { inducción }\end{array}$} & $\begin{array}{l}\text { De } 1 \text { a } 2 \text { años } \\
\text { Referendariat }\end{array}$ & No hay & No hay & $\begin{array}{l}\text { De } 1 \text { a } 2 \\
\text { años }\end{array}$ & No hay \\
\hline
\end{tabular}

Fuente: Elaboración propia a partir de Eurydice $\left({ }^{(a)} 2009,{ }^{(b)} 2012\right)$.

\section{$\underline{\text { 2.1. Instituciones formadoras y nivel de certificación mínimo exigido }}$}

Como análisis general de esta primera categoría observamos una cierta semejanza en los países seleccionados en lo que a instituciones formadoras y al nivel de certificación exigido se refiere -a excepción de Inglaterra-. Sin embargo, como veremos a continuación, con diferentes y significativos matices. En concreto, y esto en coherencia con las recomendaciones de la Unión Europea, la FIPES se realiza mayoritariamente en instituciones universitarias y con la exigencia de un Máster como certificación mínima. En Inglaterra, de forma coherente con la flexibilidad y autonomía propia de la tradición anglosajona, no existe una definición clara de estos dos aspectos fundamentales de la organización de la FIPES. Pasamos a continuación a describir de forma más pormenorizada las diferentes propuestas.

Al igual que ocurre en el conjunto de países de la Unión Europea, la FIPES de los países seleccionados se enmarca dentro de la educación superior pero no en todos los casos son las universidades o institutos dependientes de ellas las que diseñan y desarrollan los programas de formación inicial del profesorado. Tanto en Alemania como en Finlandia y en Francia se diseñan y desarrollan los programas de FIPES en las universidades y más en concreto, generalmente, dependientes de las facultades o escuelas de educación. Aspecto este compartido también por el modelo español.

En el modelo finlandés lo que destaca especialmente es la conexión establecida entre las facultades de educación con las del resto de disciplinas implicadas en el proceso de formación de 
los futuros docentes, especialmente de la Educación Secundaria (Eurydice, 2010b). Además, existe un alto grado de autonomía de las universidades finlandesas que tiene como consecuencia una especial diversidad en los planes de estudio de los programas de formación inicial docente, como podremos ver más adelante.

En el caso de Francia, se mantiene la organización institucional previa a 2010 (año en el que se produce la implementación de un nuevo y actual modelo de FIPES en este país), lo que supone la existencia de los Institutos Universitarios de Formación de Profesorado (IUFM) -inscritos en las diferentes universidades- que desarrollan programas formativos de nivel de Máster con una finalidad marcada de carácter didáctico-pedagógico. Sigue existiendo también la alternativa de formarse en los Centros Privados de Formación Educativa (CFPP), que son equivalentes a los IUFM (EURYDICE, 2010c).

Por último, en el caso actual de Inglaterra existe una amplísima gama de instituciones dedicadas a la FIPES. Esto se debe, según señala Egido (2009) a que desde los años 80 sus futuros docentes solían formarse en instituciones de educación superior (universidades generalmente) con una amplia autonomía; pero, además, la propia evolución histórica ha supuesto una definición cada vez escasa y, por tanto, una mayor presencia de instituciones no universitarias, con programas formativos muy variados. La característica común es que todas ellas deben estar acreditadas por la Teaching Agency ${ }^{5}$ (TA) como proveedoras de programas que conducen a la obtención del Qualified Teacher Status (QTS) -título imprescindible que todo futuro docente debe obtener para poder ser contratado por los centros educativos-. Para su acreditación las instituciones han de cumplir una serie de requisitos o estándares impuestos por la propia TA y la Secretaría de Estado. El sistema de acreditación de las instituciones que imparten programas de formación docente se organiza a partir de tres grupos concretos de dimensiones, y estos a su vez en un total de 22 indicadores o estándares (TDA, 2008): requisitos de ingreso para los estudiantes a los programas (6 indicadores); requisitos de las modalidades de formación y su evaluación ( 9 indicadores); y mecanismos para asegurar la calidad en las instituciones (7 indicadores).

En relación con los aspectos relacionados con la certificación mínima exigida, en términos generales, a excepción de once países europeos (entre los que se encuentra Inglaterra), la FIPES requiere la certificación mínima de Máster para su ejercicio profesional (EURYDICE, 2012). Además, en la mayoría de los países europeos, el periodo de formación suele durar entre cuatro o cinco años. En el caso de los países seleccionados, vemos como esta tendencia se cumple en Alemania, Finlandia, Francia y España. Como ya se ha indicado, en el caso de Inglaterra también hay posibilidad de que los profesores realicen titulaciones de nivel de Máster pero, en el modelo inglés no se considera el nivel de certificación como un elemento esencial sino que se opta por el concepto de acreditación externa: el denominado QTS otorgado conforme a los criterios

\footnotetext{
${ }^{5}$ La TA es heredera de la Council for the Accreditation of Teacher Educación que desde su creación, en 1984, comienza a implantar la regulación de los programas de formación inicial de los docentes en Reino Unido. Agencia que desde su origen hasta la actualidad tiene carácter estatal y se centra en el control de la calidad del profesorado inglés, de cómo llegar a serlo y de acreditar a las instituciones que pueden ofrecerse como formadoras de futuros docentes. Para más información sobre esta institución se propone la consulta de su página web: http://www.education.gov.uk/get-into-teaching [último acceso 01/05/2013]
} 
establecidos por la TA, que no depende de la realización de un programa de formación concreto en institución específicas. Hay que tener en cuenta también que en Inglaterra son los centros educativos los que contratan a sus docentes haciéndolo independientemente de que hayan conseguido el QTS por unas u otras rutas de formación.

En cuanto a la extensión de programas, en los países que optan por el nivel de formación mínimo de Máster -independientemente de que el modelo sea concurrente (caso alemán) o consecutivo (caso francés o español) ${ }^{6}$-, la duración total de la formación suele ser de cinco años. Por el contrario, cuando el nivel de formación es de Grado, su duración suele ser de tres o cuatro años; tal es el caso del Graduate Teacher Program (GTP) inglés (Grado de tipo concurrente).

\subsection{Modelo estructural del programa y formación didáctico-pedagógica}

Tradicionalmente se entiende que la formación inicial docente debe incluir dos grandes bloques generales de contenidos: el relacionado con elementos disciplinares (Química, Historia, Biología...) y con cuestiones didáctico-pedagógicas (enseñanza de la disciplina, atención a la diversidad, corrientes educativas...). De la manera en que ambos bloques formativos se complementen se distinguen tradicionalmente dos modelos estructurales de la formación inicial docente: por un lado, el modelo concurrente es aquel en el que formación disciplinar y la didácticopedagógica se realizan de forma simultánea obteniendo un único título; por otra parte, el modelo consecutivo se define porque los futuros profesores realizan primero la formación disciplinar (que les supone la obtención de un título) y con posterioridad la formación didáctico-pedagógica (que les concede la cualificación que les permite ejercer como profesores). A estos dos modelos estructurales de formación tradicionales debemos añadir, desde nuestro puno de vista, el que en las últimas décadas está asumiendo un mayor protagonismo en diferentes países a nivel mundial y que nosotros denominamos modelo mixto; este se definiría como aquel en el que los modelos estructurales de formación descritos (concurrente y consecutivo) se ofrecen simultáneamente en un mismo territorio nacional.

Entre los países seleccionados para el estudio comparado, encontramos representantes de cada uno de estos tres modelos de formación inicial. En el caso de Alemania, el modelo de FIPES es de tipo concurrente. Por el contrario, tanto en España como en Francia se opta por un modelo consecutivo. Por último, en los casos de Inglaterra y Finlandia, existen ambas alternativas de formación, aunque tal y como indica el último informe de la Eurydice (2012) en Inglaterra predomina el modelo consecutivo.

Estos modelos están presentes con un grado de intensidad diferente en cada uno de estos países. Así, podemos decir que Alemania es paradigma del modelo concurrente; Francia lo es del consecutivo; e Inglaterra del mixto. Decimos esto porque en estos tres países los modelos estructurales se mantienen igual para la formación inicial de todos sus docentes, independientemente de la etapa educativa en la que finalmente vayan a ejercer su profesión. Esto es, que en Alemania, Francia e Inglaterra, todo futuro docente -independientemente de ejercer en

\footnotetext{
${ }^{6}$ La conceptualización del modelo concurrente y del modelo consecutivo se realizará en el siguiente epígrafe.
} 
Educación Infantil, Primaria, Secundaria Inferior o Superior- se formará conforme al modelo concurrente, consecutivo o mixto, respectivamente, según se establece en su país correspondiente.

Por el contrario, en Finlandia y en España los modelos de formación inicial entre los maestros de Educación Primaria y Secundaria son diferentes. En Finlandia, los maestros de Educación Primaria se forman conforme al modelo concurrente mientras que los profesores de Educación Secundaria pueden hacerlo también por el consecutivo (modelo mixto). En el caso de España, las diferencias entre la formación de los docentes de una y otra etapa están todavía más marcadas ya que se define un modelo concurrente para los maestros de Educación Infantil y Primaria y una única vía de formación para los profesores de Educación Secundaria conforme al modelo consecutivo.

A continuación, nos ocuparemos de los aspectos relacionados con la presencia de la formación didáctico-pedagógica definida en el conjunto de los planes de estudios. En general, los programas de FIPES conforme al modelo concurrente suelen asociarse con una mayor duración de la formación didáctico-pedagógica (superior al 30\%); mientras que el modelo consecutivo se caracteriza principalmente por tener una mayor carga de formación disciplinar en el conjunto del plan de estudio (nunca la formación didáctico-pedagógica supera el 40\%).

En el caso de los países seleccionados, tal y como se muestra en la tabla 1, existe una diversidad muy amplia en el porcentaje mínimo de formación didáctico-pedagógica. Coherente con la información general descrita con anterioridad sobre el conjunto de Europa, Alemania, cuyo modelo estructural es el concurrente, es el país donde es mayor el porcentaje mínimo de formación didáctico-pedagógica (57\% del conjunto de la parte teórica de los programas). En los otros cuatro países incluidos en el estudio, los porcentajes mínimos se sitúan entre el $20 \%$ y $25 \%$. En el caso de Finlandia e Inglaterra, conviene indicar que este porcentaje puede ser muy variable en función de los programas, especialmente entre los consecutivos y los concurrentes: la presencia de formación didáctico-pedagógica en estos últimos es superior, en la mayoría de los casos, a la de los consecutivos, que suelen ofrecer una menor dedicación a este tipo de formación. Recordamos que en el caso de España el porcentaje de formación didáctico-pedagógica es del $20 \%$, con la característica de que suele ser el mismo porcentaje en todos los programas ya que el máster profesionalizador es de 60 ECTS en todos los casos y el grado disciplinar que lo antecede son de 240 ECTS. En Francia, teniendo en cuenta la actual reforma, un profesor de Educación Secundaria puede enfrentarse por primera vez a la docencia sin haber realizado ningún tipo de formación inicial de tipo didáctico-pedagógico. Es suficiente con realizar un Máster, pero no necesariamente de tipo educativo. Como veremos más adelante, la apuesta actual del modelo francés es el examen de reclutamiento nacional exigido para acceder a la profesión.

En cuanto a los contenidos de los programas, en todos los países seleccionados los centros de formación del profesorado tienen una amplia libertad para decidir el tiempo que se dedica a los distintos componentes de dicha formación. No obstante, lo que suele ser más habitual es que por medio de políticas nacionales se establezca la duración del periodo de tiempo mínimo en diferentes módulos o contenidos formativos, incluida la fase de prácticas. Pasamos a continuación a describir cuestiones más precisas sobre los curricula en los diferentes países seleccionados en base a los 
propios informes que los organismos correspondientes de cada uno de ellos realizaron en 2010 para Eurydice.

En el caso de Alemania, los planes de estudio varían en función del tipo de profesorado. En el caso de los profesores de Educación Secundaria Inferior, se exige una formación teórica-práctica de, al menos, 210 créditos ( 7 semestres) y entre sus contenidos se incluye formación general en elementos de las Ciencias de la Educación, formación práctica en centros educativos, estudios disciplinares y didácticos relativos a dos asignaturas y la realización de un proyecto que demuestre la capacidad para el trabajo científico independiente (EURYDICE, 2010b). En el caso de profesores de Educación Secundaria Superior y de Formación Profesional debe ser de al menos 300 créditos conservando los mismos contenidos y módulos generales. El peso del Practicum a lo largo del proceso formativo varía ampliamente dependiendo de las universidades de manera que no es posible precisar el mínimo de prácticas que los estudiantes deben realizar en sus estudios. Esta indefinición se explica, por un lado, porque el modelo alemán ha optado, por un lado, por hacer prevalecer la importancia de la formación didáctico pedagógica en general -que, como ya hemos indicado, debe suponer al menos el 57\% del conjunto de la formación inicial-y, por otra parte, porque es igualmente importante la fase de cualificación en el lugar de trabajo ${ }^{7}$.

La duración de los estudios en Finlandia para los "profesores de asignatura" generalmente es de entre 5 y 6 años en la alternativa del modelo consecutivo que es el más extendido para este tipo de futuros docentes. Como ya se ha indicado, el alto grado de autonomía de las universidades provoca una diversidad en el diseño de sus planes de estudio que no permite presentar un curriculum único de formación de profesores de Educación Secundaria en las diferentes universidades. No obstante, sí existen algunos principios generales que han sido seguidos por todas las instituciones encargadas de la formación del profesorado. Esto se debe principalmente a las recomendaciones del Ministerio de Educación y los acuerdos de los Decanos de las Facultades de Educación que trabajan de manera continua y coordinada. El Ministerio de Educación tiene plena confianza en los departamentos y facultades involucradas en la formación del profesorado (MEISALO, 2007). Sin embargo, de los 300 créditos mínimos de formación, al menos 60 deben ser de tipo didáctico-pedagógico de los que un mínimo de 20 han de ser prácticos. El Practicum se lleva a cabo en las escuelas asociadas; elemento característico del sistema de FIPES en Finlandia. Niemi y Jakku-Sihvonen (2009) destacan, entre otros elementos, el hecho de que los centros educativos donde se realizan las prácticas son especialmente seleccionados (una parte de esas prácticas se desarrolla en Escuelas de Excelencia, cuya titularidad es la Universidad), con tutores profesionales igualmente elegidos. El período de prácticas comienza, en el caso de los modelos concurrentes desde el primer curso de formación.

En el caso de Francia, tal y como ya se ha expresado en páginas precedentes, no existe actualmente necesidad de que los futuros profesores de Educación Secundaria realicen ningún programa concreto de formación didáctico pedagógica sino que su periodo de formación inicial se reduce a la realización de un Grado de 180 ECTS y un Máster de 120 ECTS. Como adelantábamos anteriormente, el gobierno francés ha iniciado una política que gira en torno a un examen nacional competitivo de reclutamiento del personal docente. Una última normativa ministerial publicada por

${ }^{7}$ La conceptualización de este término se realizará más adelante, en el epígrafe 2.3.2 del presente trabajo. 
el Gobierno de Francia (nº 2011-157 del 14 de septiembre 2011) alienta a los IUFM que ofrezcan a los estudiantes la posibilidad de realizar unos cursos de preparación explícita para el citado examen y fortalecer así los aspectos profesionales de la formación universitaria.

Por último, en Inglaterra, coherente con su modelo abierto de formación inicial docente, no se especifica un plan de estudios aunque sí que es prescriptivo que todos los programas cumplan con una normativa referente a la admisión, la formación y la gestión del programa (TDA, 2008). Esta normativa presta atención a las competencias y objetivos de formación que han de adquirir los estudiantes del programa: atributos profesionales, trabajo en equipo, conocimientos disciplinares y pedagógicos... De esta manera, en lo que a formación didáctico-pedagógica se refiere, en los programas concurrentes de grados de 4 años (GTP) se requiere al menos un $25 \%$ de ella y un mínimo de 32 semanas en cuanto a las prácticas. En el modelo consecutivo, con el título de Máster (Postgraduate Certificate in Education - PGCE), se especifica que las prácticas sean, al menos, de 24 semanas. Según se indica en el informe que Inglaterra realiza para la red Eurydice (2010d), las prácticas se desarrollan en varias etapas diferenciadas: una primera de toma de contacto y observación, otra de gestión de grupos de alumnos y unas últimas sesiones finales en las que el profesor asume la docencia de todo un grupo y una disciplina determinada durante un periodo prolongado de tiempo.

\subsection{Procesos de selección para acceder al programa de formación y a la profesión}

Hasta este momento se han abordado elementos de la FIPES relacionados, principalmente, con el programa de formación. Sin embargo, desde un enfoque de desarrollo profesional docente se hace necesario incluir también las cuestiones relacionadas con los procesos de selección de los candidatos. Así, se pueden distinguir dos momentos diferenciados para este objetivo: la selección en el acceso al programa de formación y un segundo momento relacionado con el acceso a la profesión docente, propiamente dicha. A continuación, pasamos a exponer los principales resultados de los países europeos seleccionados en estos dos aspectos.

\subsubsection{Proceso para acceder al programa de formación}

En la última década, los principales informes internacionales están poniendo de manifiesto la importancia que la selección del profesorado tiene sobre el rendimiento de los alumnos (OECD, 2005; BARBER y MOURSHED, 2007). En este sentido, el acceso al programa de formación se convierte para todo sistema de FIPES en el primer momento para realizar dicha selección. A diferencia de aspectos abordados con anterioridad, en este elemento sí que encontramos diferencias mucho más significativas entre los países seleccionados, que pasamos a comentar.

En el caso alemán el acceso principal a los programas es el propio del acceso general a la universidad, consistente en la superación de la prueba denominada Abitur. Este examen, que suele identificarse con la prueba de selectividad del caso español, se realiza en los propios centros escolares donde se finaliza en Gymnasium y consiste en unas pruebas orales y escritas, sobre contenidos de los estudios que han finalizado, diseñadas por organismos de los diferentes Länder. 
Superar esta prueba supone la obtención del Hochschulreife (aptitud para el acceso a la Educación Superior).

En el caso de Finlandia, y al igual que ocurre en Alemania, para los estudiantes que optan por el modelo concurrente, el acceso se produce con la finalización exitosa de la Educación Secundaria pero se acompaña de un examen escrito, una prueba de aptitud y una entrevista personal realizada por las universidades correspondientes bajo la supervisión de algunas medidas a nivel estatal. Por el contrario, aquellos candidatos que van a acceder a los programas que siguen el modelo consecutivo, pueden hacerlo tras finalizar los estudios de grado (de 3 años de duración en el caso de Finlandia). Para acceder a estos programas de posgrado para la formación docente (Máster de 2 años), los estudiantes han de superar una prueba de aptitud y se tiene en cuenta el expediente académico. Tan solo un 17\% de los solicitantes superaron las pruebas de acceso en el 2009 (EURYDICE, 2010b). Estos datos confirman el elemento tan característico del sistema finlandés en lo que a exigencia en el acceso se refiere. Un proceso complejo y exigente de selección para acceder al programa de formación es la apuesta más decidida del sistema de FIPES en Finlandia; lo que explica los buenos resultados de rendimiento de sus estudiantes según numerosos informes internacionales de reconocido prestigio (OECD, 2005; BARBER y MOURSHED, 2007).

Como ya se ha indicado con anterioridad, Francia es el país que mejor representa el modelo consecutivo como única vía de formación del profesorado de todas las etapas educativas. La principal característica del actual modelo francés es que no existe la obligación de cursar un programa de formación didáctico-pedagógica específico para acceder a la profesión docente, sino, simplemente, realizar un Máster de dos años de duración (el cual no tiene por qué ser necesariamente alguno de los antiguamente desarrollados en los Institut Universitaire de Formation des Maîtres -IUFM-). Hasta el curso 2010, para acceder a los másteres de carácter didácticopedagógico impartidos por los IUFM se exigía: un buen nivel en las materias de especialidad didáctica elegidas por el candidato, contar con las capacidades esenciales para la enseñanza (EURYDICE, 2010c), y estar en posesión de un título universitario de Grado de tres años de duración (o licenciatura en los planes antiguos) de cualquier disciplina. La última reforma al respecto, ha sustituido la formación didáctico-pedagógica por un proceso de selección que consiste en la superación de un examen competitivo nacional al cual es posible presentarse tras haber realizado estudios de Máster. En Francia, es la prueba misma la que define el modelo -si se puede definir como tal- de FIPES. En este sentido, podemos indicar que Francia ha apostado por una formación inicial basada en la definición de un perfil competencial y su adquisición por parte de los candidatos. El programa formativo (Máster) deja de ser lo esencial para la selección de los futuros docentes.

Por último, en Inglaterra, al igual que sucedía en Finlandia, los requisitos para acceder a los programas de formación docente, dependen de la vía de formación elegida (modelo concurrente a través del gado o consecutivo por medio del posgrado). Si se opta por alguno de los programas de tipo concurrente, los requisitos de admisión son los propios del acceso a los estudios universitarios en los que la selección de los estudiantes se realiza por parte de las instituciones de Educación Superior correspondientes. En el caso de optar por un modelo de formación consecutivo, los criterios varían en función de la institución de formación. Sin embargo, como norma general, es 
preceptivo que todos estos programas exijan a sus candidatos (TDA, 2008): superar una entrevista diseñada para evaluar la idoneidad, contar con la capacidad intelectual necesaria, leer con eficacia y comunicarse con claridad en inglés, no tener antecedentes penales, estar registrados provisionalmente en el Consejo General de Enseñanza y cumplir una serie de requisitos de salud y capacidad física para enseñar.

\subsubsection{Proceso para acceder a la profesión y fase de cualificación en el lugar de trabajo}

En algunos países europeos comienza a ubicarse como modalidad para realizar la selección al acceso a la profesión la denominada fase final de cualificación en el lugar de trabajo, o de iniciación a la vida laboral. Esta puede considerarse como la última fase de la formación inicial del profesorado. Su importancia reside en su dimensión de apoyo y supervisión, así como de evaluación formal de la competencia profesional (EURYDICE, 2009). Este mismo informe indica que la fase de cualificación en el lugar de trabajo debe caracterizarse, al menos, por lo siguiente:

- Debe tratarse de un periodo obligatorio.

- Los candidatos no deben ser docentes.

- Los candidatos forman parte de la plantilla del centro.

- Los candidatos suelen recibir una remuneración.

- Debe tener una duración de, al menos, un año.

Esta fase ha comenzado a implantarse cada vez en más países europeos llegando hasta un total de 13 según el último informe realizado por Eurydice (2012). Entre los países seleccionados para nuestro estudio, tan solo se propone en los sistemas alemán e inglés. En ambos países esta fase se realiza tras la formación teórico-práctica y se entiende como un programa de apoyo estructurado y obligatorio para poder optar a la contratación como profesores. Durante todo el proceso, estos "profesores candidatos" llevan a cabo la mayoría de las tareas habituales de los docentes con experiencia. En ambos países, es especialmente relevante la figura del tutor y la evaluación formal que reciben de las competencias docentes que se espera hayan adquirido.

A pesar de las semejanzas señaladas, existen algunas diferencias entre los sistemas alemán e inglés que conviene resaltar. En primer lugar, la duración de esta fase en el modelo alemán es de dos años completos mientras que en Inglaterra es suficiente con un año. En Alemania, al denominado Vorbereitungdienst -servicio preparatorio- le antecede la realización de un primer examen nacional que da acceso a esta fase de cualificación en el lugar de trabajo. Una vez concluido este, sucede un segundo examen de Estado que los candidatos deben superar pero que no garantiza el puesto de profesor (EURYDICE, 2010a).

En Inglaterra, la capacitación docente se obtiene después de un programa de inducción que dura un curso escolar. Consiste en un programa de prácticas que se lleva a cabo en determinados colegios asociados, los cuales establecen previamente un convenio con las instituciones formadoras. Los programas de inducción son individualizados, los candidatos no superan el $90 \%$ del horario de otros profesores de la escuela y si no se completa satisfactoriamente no pueden optar al empleo de profesor (EURYDICE, 2010d). 
En cuanto a los otros tres países seleccionados (Finlandia, Francia y España), no incluyen fase de cualificación en el lugar de trabajo. En el modelo finlandés, el proceso de selección se centra en los momentos previos al periodo de formación inicial, mientras que en Francia y España se opta por realizar la selección después de recibir la formación en las universidades. Además, en el caso de Finlandia, las políticas que se desarrollan tras finalizar la fase de formación inicial, se centran en los mecanismos de apoyo a los profesores noveles en sus centros. Estas medidas son especialmente eficaces debido a la autonomía que tienen los institutos para ayudar a sus nuevos profesores a incorporarse a la comunidad educativa (EURYDICE, 2012).

\section{CONCLUSIONES}

A la luz de los resultados expuestos sobre la política educativa de la Unión Europea y de países específicos en materia de FIPES, consideramos pertinente ofrecer a continuación los siguientes elementos de discusión y sugerencias de mejora.

El análisis documental de la normativa emanada desde la Unión Europea en materia de formación inicial docente invita a pensar en la necesidad de crear marcos de armonización dentro de este ámbito. Estudios internacionales, y la propia Unión Europea, han manifestado reiteradamente la importancia de cuidar los elementos relacionados con la preparación de los futuros docentes. Así pues, consideramos que normativas más específicas y acordadas por parte de los Estados miembros podría ser una medida adecuada. Esto exigiría, sin duda, que la Unión Europea fuera quien liderase esta propuesta pero con el acuerdo de los Estados miembros y la suficiente flexibilidad para que sean estos últimos los que las adapten a sus realidades y tradiciones educativas.

Siendo más específicos en el análisis, y teniendo en consideración resultados del estudio comparado de los cuatro países seleccionados, este proceso de armonización podría comenzar con la definición de un perfil competencial docente europeo. En este sentido, el proyecto del Finnish Institute for Educational Research (FIER, 2009) al que hemos hecho referencia en el artículo, representa un primer paso consistente en esta dirección. Pero es preciso avanzar. La posibilidad de tener un marco común de competencias profesionales, no solo nos permitiría situar el desempeño docente sino, también, reconocer la relevancia que el nuevo paradigma del aprendizaje por competencias tiene para orientar el diseño y desarrollo de programas de formación -inicial y permanente- del profesorado. Esto, sin tener en cuenta otras grandes ventajas como serían las relacionadas con los intercambios, la movilidad, las redes de aprendizaje... Estos marcos compartidos de competencias son especialmente relevantes en estos momentos, pues representan una forma muy adecuada para poner en marcha una formación inicial del profesorado orientada hacia la adquisición progresiva de desempeños profesionales.

A su vez, esta propuesta entronca perfectamente como un posible punto de partida clave para orientar adecuadamente los programas de formación inicial. Este relevante periodo está configurado por diversos procesos cuya importancia reside en la visión global del desarrollo profesional docente. Entre ellos destacamos la selección en el momento del acceso al programa de formación; el programa formativo teórico-práctico (con especial interés en la formación didácticopedagógica y la disciplinar y en la relevancia del Practicum y de cómo este se desarrolla); la 
selección en el momento del acceso al ejercicio de la profesión docente; y la inserción en el lugar de trabajo.

A la luz del estudio comparado, identificamos al menos cinco aspectos que podrían ayudar en la mejora de la FIPES en nuestra realidad nacional, y también para la del conjunto de los países de la Unión Europea.

El primero de ellos haría referencia a la necesidad de flexibilizar los modelos estructurales. Hemos comprobado como en Europa existe un amplia diversidad de formas de estructurar la FIPES, pero que la tendencia parece orientarse hacia una formación que hemos denominado de modelo mixto. Parece evidente que las diferentes tradiciones históricas, culturales, sociopolíticas y educativas de los países condicionan la estructuración de esta primera fase de formación de los docentes pero en ningún caso esto debería suponer limitar la calidad de los programas formativos de los futuros docentes. La apuesta por un único modelo (concurrente o consecutivo), como es el caso de España, podría verse flexibilizada por nuevas propuestas que permitieran mayor diversidad y tendencia hacia los denominados modelos mixtos. Es importante indicar a este respecto que esta propuesta exige igualmente una definición previa $-\mathrm{y}$ revisable- del denominado perfil competencial docente. Este se convertiría en el horizonte a conseguir por parte de todos los programas de formación docente, más allá de sus características o estructuraciones específicas.

Como segundo elemento, y teniendo en consideración lo expuesto en el párrafo anterior, se entiende que las propuestas de la Unión Europea en torno al nivel de certificación mínimo exigido para el ejercicio profesional docente es el adecuado. El nivel de Máster exigido para todos los docentes, como nos muestran los resultados de Finlandia, supone, principalmente, un valor añadido en lo relacionado con el prestigio social de la profesión y no sólo por solicitar una certificación de mayor nivel sino porque esta supone también un reconocimiento académico y, por tanto, un status a la docencia como profesión especializada (compleja, exigente...). Esta propuesta tiene además la ventaja de flexibilizar la manera de organizar la formación inicial del docente y, por tanto, el desarrollo de modelos estructurales mixtos.

Por el contrario, la cuestión sobre la que existiría una mayor controversia a la hora armonizar la formación inicial docente en la Unión Europea residiría en la finalidad de los programas y el peso de la formación didáctico-pedagógica y la disciplinar. Este tercer elemento resulta más controvertido en el caso del profesorado de Educación Secundaria pues, tradicionalmente, esta etapa ha sido destinada a la especialización académica que exigía que prevaleciera la formación disciplinar. Sin embargo, la universalización de la Educación Secundaria a partir de los años 60 en Europa, supone reconocer que los docentes ya no solo tienen que saber mucho de las materias que enseñan sino que también deben saber cómo transmitirla, como atender a la diversidad, a las familias, trabajar con otros... Es esta evolución la que ha llevado necesariamente a la profesionalización de la docencia. Así, la formación didáctico-pedagógica parece cada vez más necesaria en el profesorado de Educación Secundaria. Sin embargo, existe todavía hoy cierta resistencia al cambio y formas de organizar la FIPES, como el modelo consecutivo, son todavía ejemplo de un predominio de la formación disciplinar frente a la didáctico-pedagógica en algunos países, como España. 
Más allá de esta cuestión, y como cuarto aspecto a considerar, parece importante remarcar los aspectos relacionados con la fase de prácticas en los planes de estudio. Los informes internacionales y las propias políticas nacionales y supranacionales europeas apuntan en la misma línea: los programas formativos deben girar en torno a una adecuada fase de prácticas donde los estudiantes despliegan, y mejoran, sus competencias como docentes. Sin embargo, nos encontramos todavía hoy con una gran diversidad y falta de criterio en torno a esta cuestión. Si todos compartimos la importancia teórica -y el sentido- de esta fase de prácticas en los planes de estudio, este aspecto debería poder ser otro elemento sobre el que desarrollar algún tipo de propuesta supranacional que permitiera una mayor armonización en la Unión Europea. En este aspecto, al igual que en el nivel de certificación, los modelos consecutivos, de nuevo, suelen ofrecer menos posibilidades de realizar prácticas en centros educativos, en comparación con los modelos concurrentes.

Por último, a la luz de los resultados, parece necesario que reflexionemos en torno a la necesidad de mejorar los procesos de selección de los candidatos. El principal aspecto que nos enseña Finlandia a este respecto es que no es suficiente con el simple hecho de ser restrictivos, sino que se requiere el diseño de mecanismos de evaluación diversos que aseguren candidatos más adecuados para el ejercicio de la docencia, y respetando el principio de igualdad de oportunidades. Recordamos que esta selección se puede hacer en dos momentos principales: para acceder al programa de formación y para acceder al ejercicio profesional propiamente dicho. Como hemos visto, el proceso de selección de los docentes finlandeses se centra en el acceso al programa de formación y se realiza mediante métodos diversos y sucesivos que valoran conocimientos, aptitudes y motivaciones mediante técnicas escritas, orales y, esencialmente, prácticas. Y además de ello, las pruebas de acceso, para ser eficaces, incluyen entrevistas personales, test de aptitudes, niveles motivacionales, conocimientos... Medidas coherentes con estas orientaciones parecen ser las más adecuadas y las que deben proponerse como políticas de los estados. En relación con los procesos de selección de los candidatos a la profesión recordamos que en países como Alemania o Inglaterra, donde no hay una selección exigente de acceso al programa formativo, para asegurar el inicio de la adquisición de competencias docentes se ha optado por enfatizar la fase de cualificación en el lugar de trabajo. Hay otros países, como Francia o España, que para el acceso a la docencia optan por un proceso de inducción cualitativamente diferente, basado en la superación de un examen principalmente de conocimientos memorístico y declarativo. En este sentido, la dedicación de, al menos, un año completo de despliegue de las competencias profesionales en el puesto de trabajo consideramos que sería otra medida fundamental sobre la que los estados podrían pivotar las políticas de formación inicial del profesorado.

Todos los aspectos desarrollados hasta el momento parecen de vital importancia si tenemos en consideración la relevancia de la formación inicial del profesorado en el rendimiento de los alumnos y la calidad de la educación. Así, consideramos que estas claves interpretativas pueden ser de ayuda en la reflexión académica y política. No obstante, se hace imprescindible continuar aumentando las evidencias empíricas a este respecto y esto, para que influyan en la toma de decisiones políticas nacionales ajustadas a su realidad socioeducativa. Los responsables políticos, empujados por el conjunto de la comunidad educativa (alumnos, familias, profesores, equipos 
directivos, sindicatos...) deben implicarse para desarrollar medidas que favorezcan la mejora de la educación. Y esto exige reconocer la necesidad de cuidar al profesorado y su formación inicial.

\section{REFERENCIAS BIBLIOGRÁFICAS}

BARBER, M. y MORUSHED, M. (2007): How the World's Best-Performing School Systems Come Out On Top. (London, McKinsey \& Company, Social Sector Office).

CONSEJO (1996a): Resolución del Consejo de 6 de mayo de 1996 relativa a los software educativos multimedios en el campo de la educación y de la formación. Diario Oficial de la Unión Europea, serie C, no 195, 6 de julio de 1996, p. 8.

CONSEJO (1989a): Decisión del Consejo de 28 de julio de 1989 por la que se establece un programa de acción para promover el conocimiento de lenguas extranjeras en la Comunidad Europea (Lingua). Diario Oficial de la Unión Europea, serie L, nº 239, 16 de agosto de 1989, p. 24.

CONSEJO (1989b): Resolución del Consejo y de los ministros de educación reunidos en el seno del Consejo de 22 de mayo de 1989 relativa a la escolarización de los niños gitanos e itinerantes. Diario Oficial de la Unión Europea, serie C, nº 153, 21 de junio de 1989, p. 3.

CONSEJO (1990a): Resolución del Consejo y los ministros de Educación reunidos en Consejo, de 31 de mayo de 1990, relativa a la integración de los niños y jóvenes minusválidos en los sistemas educativos ordinarios. Diario Oficial de la Unión Euroepa, serie C, nº 162, 3 de julio de 1990, p. 2.

CONSEJO (1990b): Conclusiones del Consejo y de los ministros de Educación reunidos en Consejo, de 31 de mayo de 1990, sobre el fomento de la igualdad de oportunidades educativas entre los sexos en la formación inicial y la formación permanente de los profesores. Diario Oficial de la Unión Europea, serie C, n 162, 3 de julio de 1990, p. 6.

CONSEJO DE EDUCACIÓN (2001): Informe del Consejo "Educación" al Consejo Europeo Futuros objetivos precisos de los sistemas de educación y formación [5680/91EDU 18].

CONSEJO EUROPEO (2000): Conclusiones de la presidencia del Consejo Europeo de Lisboa de 23 y 24 de marzo. Recuperado de:

http://www.europarl.europa.eu/summits/lis1_es.htm

DALE, R. y ROBERTSON, S. (2006): The case of the UK: Homo sapiens Europaeus vs. homo quaestuosus Atlanticus? European learning citizen or Anglo-American human capitalist?, en M. Kuhn y R. G. Sultana (Eds.): Homo sapiens Europaeus: Creating the European learning citizen. New York, Peter Lang, pp. 21-46.

DEHMEL, A. (2006): Making a European area of lifelong learning a reality? Some critical reflections on the European Union's lifelong learning policies. Comparative Education, 42(1), pp. $49-62$.

EGIDO, I. (1996): La política educativa de la Unión Europea: principales etapas de desarrollo. Tendencias pedagógicas, 2, pp. 19-29.

EGIDO, I. (2009): La profesión docente en la Unión Europea: tipologías, modelos, políticas. En M. de Puelles (Coord.), Profesión y vocación docente. Presente y futuro (pp. 201-227). Madrid: Biblioteca Nueva-Escuela Julián Besteiro. 
ERTL, H. (2006): European Union policies in education and training: the Lisbon agenda as a turning point? Comparative Education, 42(1), pp. 5-27.

EUROPEAN COMMISSION (2005): Common European Principles for Teacher Competences and Qualifications. Brussels, Directorate-General for Education and Culture.

EURYDICE (2009): Cifras clave de la educación en Europa 2009. Bruselas, Eurydice.

EURYDICE (2010a): Organization of the education system in Germany 2009/10. Brussels, Eurydice.

EURYDICE (2010b): Organization of the education system in Finland 2009/10. Brussels, Eurydice. EURYDICE (2010c): Organization of the education system in France 2009/10. Brussels, Eurydice.

EURYDICE (2010d): Organization of the education system in the United Kingdom: England, Wales and Northern Ireland 2009/10. Brussels, Eurydice.

EURYDICE (2012): Key data on Education in Europe 2012. Brussels, Eurydice.

FIELD, J. y MURPHY, M. (2006): Governance and the learning citizen: Tensions and possibilities in the shift from national and post-national identities, en M. Kuhn y R. G. Sultana (Eds.), Homo sapiens Europaeus: Creating the European learning citizen. New York, Peter Lang, pp. 69-82.

FIER (2009): Education and Training 2010: Three studies to support School Policy Development (Tender $N^{\circ}$ EAC/10/2007). Lot 2: Teacher Education Curricula in the EU. Final Report. Jyväskylä, University of Jyväskylä.

GARCÍA GARRIDO, J. L. (1991): Fundamentos de la educación comparada. Madrid, Dykinson.

KWIEK, M. (2004): The Emergent European Educational Policies under Scrutiny: the Bologna Process from a Central European perspective. European Educational Research Journal, 3(4), pp. 759-776.

OECD (2005): Attracting, Developing and Retaining Effective Teachers - Final Report: Teachers Matter. Paris, OECD.

PHILLIPS, D. y ERTL, H. (2003): Implementing European Union education and training policy. A comparative study of issues in four Member States. Dordrecht (Holland), Kluwer.

TDA. Training and Development Agency for Schools (2008): Professional Standards for Teachers. Why sit still in your career? (http://www.merton.gov.uk/learning/trainingdevelopment/professional standards for teachers.pdf [último acceso 01/12/2012]).

TUSCHLING, A., and ENGEMANN, C. (2006): From Education to Lifelong Learning: The emerging regime of learning in the European Union. Educational Philosophy and Theory, 38(4), pp. 451-469.

VALLE, J. M. (2009): Política educativa de la Unión Europea: evolución e hitos contemporáneos, en M.J. Martínez Usarralde (coord.): Educación Internacional. Madrid, Tirant lo Blanch, pp. 197-234. 


\section{PROFESIOGRAFÍA}

\section{Jesús Manso Ayuso}

Doctor en Educación por la Universidad Autónoma de Madrid en la categoría de Personal Docente e Investigador en Formación por su contrato de la ayuda de Formación del Profesorado Universitario (FPU) del Ministerio de Educación. Coordinador de áreas del Grupo de Investigación sobre Políticas Educativas Supranacionales y miembro del equipo directivo de la Asociación de Jóvenes Investigadores de Teoría e Historia de la Educación. Maestro en la especialidad de Educación Especial y licenciado en Psicopedagogía. Entre sus méritos académitos destaca la Beca de Excelencia de la Comunidad de Madrid (2007-2008) y el Premio Nacional a la Excelencia Académica del Minsiterio de de Educación. Entre sus líneas de investigación y publicación está la formación del profesorado, la política educativa y la educación supracionacional. Datos de contacto: E-mail: jesus.manso@uam.es

\section{Javier M. Valle López}

Doctor en Ciencias de la Educación. Premio Extraordinario de Doctorado y I Premio Nacional de Tesis Doctorales en Educación Comparada «Pedro Roselló». Profesor Titular en la Universidad Autónoma de Madrid, donde imparte «Políticas Educativas de la Unión Europea» y «Política educativa: la visión Supranacional». Especialista en Políticas Educativas de Organismos Supranacionales (principalmente Unión Europea). Ha realizado investigaciones para organismos tales como la UNESCO, la OEI o EURYDICE, dando como resultado numerosas publicaciones en libros y en revistas científicas nacionales e internacionales. Una de las más destacadas es «La Unión Europea y su política Educativa», en dos tomos (Madrid, MEC, 2006). Actualmente es Director del Grupo de Investigación Reconocido por la UAM sobre «Políticas Educativas Supranacionales». Su actividad científica se proyecta también en numerosas conferencias internacionales. Ha sido Jefe de estudios de Psicopedagogía en la UPCO y Vicedecano de Ordenación Académica en la UAM. Asesor externo de la Unidad Española de EURYDICE. Forma parte del equipo TEAM-EUROPA, de conferenciantes expertos en Unión Europea, de la Representación Española de la Comisión Europea.

Fecha de recepción: 23 de diciembre de 2012.

Fecha de revisión: 28 de enero de 2013 y 22 de mayo de 2013.

Fecha de aceptación: 14 de junio de 2013. 